\title{
Application of Layout Design Theory in the New Media Era
}

\author{
Jinxia Wang * \\ Shaanxi Fashion Engineering University, Xianyang, Shaanxi, China \\ *1754354519@qq.com
}

\begin{abstract}
Under the impact of new media on traditional media, the layout design has undergone new changes. The application of visual psychology in the layout design of new media is focused on. Through the knowledge of visual psychology combined with the excellent cases of relevant layout design, the methods and techniques of new media layout design are analysed. Thus, the layout design techniques based on visual psychology and visual psychological feelings in layout design are summed up. Under the new media environment, paying more attention to the layout design brings about the psychological experience and visual beauty for people. Therefore, layout design has become one of the essential basic skills of modern design. The research on the application of new media layout design based on visual psychology has more important guiding significance.
\end{abstract}

Keywords: new media; layout design; digital technology; webpage design.

\section{Introduction}

"Layout design" plays a very important role in the process of visual communication, as a regular form of graphic language communication. Most of the audience just simply browse, but they can design from the visual psychological perspective of the audience and give people different visual feelings. Especially in Europe, because of the influence of social and cultural environment, designers can often grasp this "visual effect" in the picture, also known as "propaganda effect". Taking the Finnish graphic designer Kiosti Wallis as an example, he adopted the method of arranging a large number of pictures as the background of the layout in the layout design, and then used the layout law to design a new type of work. The indispensable elements of pictures and characters in layout design, after reasonable arrangement, will make the text become sequenced. As a result, the audience can quickly grasp the theme of the layout from a simple visual browsing. Thus, it alleviates the visual fatigue of the public, and the layout will be more readable, highlighting the cultural atmosphere [1].

\section{Literature Review}

Visual psychology is a discipline that explores the connotation of perception, which contains rich contents. As an independent subject, visual psychology is closely related to other disciplines, including physical optics, eye and brain anatomy and physiology, visual physiology, visual learning, vision and art. Rudolph Arnheim, a professor of art psychology at Harvard University, mentioned in his book Visual Thinking that one of the aims of visual thinking is to systematically analyze the effects of vision in order to guide people's vision and restore its functions. He elaborated on the early germination of visual thinking from the separation of perception and thinking, distrust of feeling, Plato's distinction of two kinds of reason, and Aristotle's exploration of "top-down" and "bottom-up" [2].

Donald A. Norman said in the book Design Psychology: the human brain uses symbols, symbolic structure and symbolic manipulation to characterize and explain the external world. "Symbol" is the carrier of information. It can be seen that these psychological representation represents the information stored in the outside mind [3].

Wang Lingzhong analyzed and decomposed the visual psychology in his book Art Effect and Visual Psychology from the following aspects: the basis of sharing, the natural way of art, the dynamic composition of artistic psychological effect, and the decomposition and verification of formal effect. When it comes to the constant exchange of information between the inner and outer worlds, this structure explains the nature of "information exchange" relationship between people and the external environment [4]. 
Richard Gregory, a famous professor of experimental psychology at Cambridge University, made outstanding contributions to visual psychology. He had an outstanding insight into visual psychology. He divided visual psychology into perception, neuropsychology, experimental psychology, cognitive science, artificial intelligence, and scientific research methods. Therefore, it is known that visual psychology is a complex subject. Especially based on visual psychology, new media format can dig out more valuable "visual emotional needs", thus bringing consumers a new sense of visual experience in the visual psychological perspective [5].

Japanese author Akayama Sachiko's Network Design Principles systematically described the related knowledge of art design and technology application, which played a great role in promoting the development and progress of layout design [6].

\section{The Application of Visual Psychology in the Layout Design of New Media}

\subsection{Layout Design in New Media}

The layout design of new media is mainly based on digital information technology, which makes great changes in the speed, efficiency and mode of information dissemination. With the rapid development of science and technology, the application of digital technology and electronic screen in new media has greatly improved the diversity of layout and the variety of visual language. At the same time, it is also an inseparable part of people's lives, showing a broader space for development. At present, this kind of design work is one of the more popular work, providing a great development direction and space for the development of layout design [7].

With the wide spread of new media technology, interactive forms of information dissemination are becoming more and more diverse. Interactive formats, based on a digital media, are applied to the layout design of computer interaction technology. Finally, they are presented in the form of electronic screen. Its purpose is that the browser can process and operate according to its interaction on the layout, so that the interaction between the browser and the information can be generated. The direct interaction between the experiencer and the works makes the experiencer seem to be in it, making the interaction and experience become the main way of layout design. Relying on the people-oriented theme, it can make the structure and shape of the traditional layout changed, thus realizing the viewer to experience different forms such as touch, space movement, voice and other new forms to trigger format conversion, so as to complete the instructions of browsers. Therefore, the art form of layout design in the new media can not only satisfy the colour, graphics, and text, but also need to consider the psychological needs of the viewer, and consider the interaction between the device and the experiencer, so that the design of the interface is more humanized.

\subsection{Advantages and Characteristics of New Media Layout Design}

In the new media environment, image information has become the core strength of the layout of new media. In today's new media layout design, pictures, as the main layout form of design elements, are gradually increasing. With the popularity of optical fibre broadband and $4 \mathrm{G}$, the speed of receiving messages has been greatly accelerated. The greatest advantage of pictures is that they can transcend the power of the text itself and directly bring visual perception to the audience. Therefore, the status of pictures in the layout of new media should not be underestimated. In new media, images consist of static and dynamic images, including photography, illustrations, icons and other categories. Dynamic images mainly include FLASH videos, GIF and so on. In traditional layout design, pictures have their own rules of arrangement. For example, when there are dialogue scenes in the content of the page, character should be arranged face-to-face. In the overall layout, it is supposed to pay attention to the relationship between words and characters, the relationship between words and objects, and the combination of pictures. In the picture, the dynamic direction relations and changes of scenes and characters should also be focused on. Traditional layout design technology is mainly used for static image layout design. Now, some key principles of new media dynamic image layout design are summarized: effective classification of prominent themes in the layout content, and choice of a reasonable layout of dynamic image, so that its layout content is more perfect. 


\subsection{Theoretical Basis of Visual Psychology in Layout Design}

In view of visual psychology, it is closely related to design. Back in the early 20th century, the Bauhaus Institute of Design in Germany was the first institution to study and contribute most to visual psychology, and its design always has forward-looking characteristics. To cater to the development trend of the times, it also advances with the times to put forward related research on the "visual psychology", which contributes more research theories and monographs for the study of visual psychology. It caters to the development of visual psychology design needs and provides sufficient rational materials for visual psychology related cognition. When it comes to visual psychology, Gestalt psychology has to be mentioned. In the work Art and Visual Perception written by American esthetician Rudolph Arnheim, he applies "Gestalt Psychology" to visual art very well. With the help of his book Art and Visual Perception, he has laid a solid foundation for the science of artistic psychology. He applies the new discoveries and achievements of modern psychology in it, and also summarizes the corresponding visual psychological feelings under various factors. Another British art theorist, E. H. Gombrich, also has a deep knowledge of visual art psychology. His work Art and Illusion expounds that visual psychology is a kind of human ability to understand the external world actively, which includes not only the imaginative elements of objective things, but also contains the conceptual reasoning of things. The objective facts are presented as subjective impressions through visual and cortical filtering, so as to form visual effects that convey information. In their theories, the two scholars have made outstanding contributions to visual psychology, provided sufficient theoretical support for the development of art design and visual psychology to a certain extent, and also made clear planning of the development path of modern art design, which laid the foundation for the development of visual psychology.

\section{Application of Visual Psychology in the Layout Design of New Media}

New media format design is closely related to people's life, so the new media format design should stand in the audience's perspective to explore its design motive force and development trend. Therefore, visual psychology is the theoretical basis for designers to design the layout of new media. The visual psychological analysis of new media format design and the application of visual psychology in new media format design are discussed.

\subsection{Application of Visual Psychology in User Interface (UI) Web Page Design}

From the visual psychological point of view, the most attractive design guide in UI web page design that can perceive the leading position is the web page having impact on the vision.The socalled "law of visual flow", popular saying is: when browsing information pages, people habitually look at what first, then see what, which is such a law of visual browsing process. The law of visual flow is a complicated physiological process. It is not only a matter of habit, but also related to human visual physiology, thinking logic and psychological habits. It embodies the connotation of ergonomics. According to the relevant investigation and analysis, the law of visual flow is directional [8]. Generally speaking, from top to bottom, from left to right, from dynamic to static, from light to dark, from thick to thin lines, the audience prefers to receive uniform and coherent information in form and content, which reflects the guiding significance of web page information reception. Usually, the visual excitement of a web page has a strong visual impact. It is also greatly different from other elements of the page, specifically embodied as strong colour prominent and unique form.

Layout design of web page columns: web design and other types of design have a lot in common. For example, newspapers, the basic principles of design should also be followed. After being familiar with design principles and considering the particularity of the page, it is not difficult to design a beautiful and generous page consistent with human visual psychology. People familiar with webmaking may soon form a general layout in their minds, and when they get the content of a web page, they can start production directly using web-making tools. In the layout design, it is necessary to first draw a layout sketch of the publishing surface (as shown in Figure 1 below), and then refines and adjusts it repeatedly to determine the final layout. The new page is a blank sheet of paper, without 
any form, frame or practice. Use your imagination as much as possible and draw your ideas. This is the creative stage, sketching the idea with rough lines.

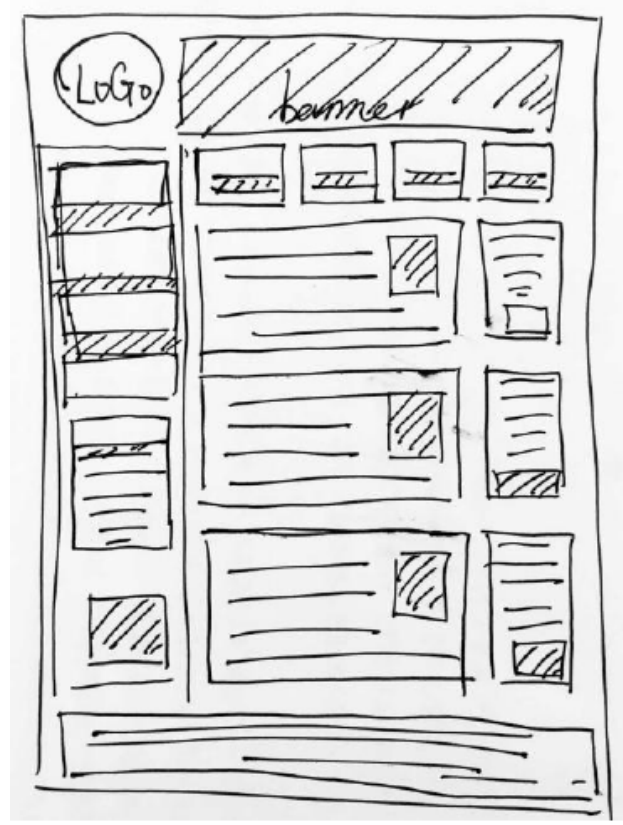

Figure 1. Web page manuscript

Visual psychology of web page layout: web design utilizes the basic visual elements of graphic design to achieve the purpose of information transmission and aesthetic appreciation. These visual elements include text, fonts, graphics and images, as well as layout. Visual elements and their combination of forms and methods, as well as web design styles are diverse. They not only meet the visual needs of web visitors, but also meet the function of information transmission on the web page. Commercial websites mainly serve consumers by browsing. All visual effects and typesetting design are based on commercial consumption. With this as a fulcrum, the relationship between image, technology application and text can be better handled. The basic elements of visual space are composed of dots, lines and planes, and also the basic language of visual expression. From the perspective of visual psychology, the layout design of web pages is to deal with these three visual relations. The dots, lines and faces in web pages are interdependent and indispensable. They complement each other, forming all kinds of visual images and changing visual space.

\subsection{Application of Visual Psychology in H5 Interface Design of Mobile Phone}

Flattening of H5 interface design: nowadays, electronic devices are constantly infiltrating people's life. Mobile phones, computers and electronic devices such as tablets are playing an increasingly important role in people's life. Smart portable devices play a particularly important role. With the advent of electronic portable intelligent devices, as a result, the visual needs of the product have gradually come into people's vision. People pay more attention to the visual experience and feelings on electronic devices, as well as intelligent interaction effects, namely UI design, which has gradually entered the scope of interface design.

H5 here is not HTML5, but a new way of information dissemination taking HTML5 as a basic code, which relies on mobile phone interface and also a form of dissemination very popular in recent years. In recent years, with the development of technology and the deepening of people's understanding of $\mathrm{H} 5, \mathrm{H} 5$ has gradually developed from the traditional form of page turning to more interactive and visual effects. As shown in Figure 5 below, the H5 uses a smooth H5 sliding interaction, and coupled with strong product planning, it is visually with gray as the main colour, strongly reflecting the $\mathrm{H} 5$ theme. The visual impact of the rapid visual sliding effect, together with the colour matching, it is unified in style and bold in design, with childhood as the theme, arousing 
the resonance of the post-80s and post-90s. The development of H5 Games has also become a new favourite of some enterprises. The design of game H5 not only needs to highlight the theme visually, but also should accord with the relevant requirements of game design and add the necessary game elements, so that users can have a better interactive experience.

Case analysis of H5 interface design: it is conducted at a stage of exploration. Gradually, to do well in H5, it is necessary to have a clear design procedure. Now, several sets of H5 production process cases are analyzed. H5 design is not only limited to the screen design, but also needs to consider the advantages and disadvantages of design from the hearing, vision, touch, interaction and other factors. It is worthy studied when to do animation switching effect, when to play H5 background music and when to produce as well as user interaction effects. Before designing H5, there should be a good idea and thinking to arrange specific music and interactive usage scenarios from the user's point of view. Therefore, to design $\mathrm{H} 5$ well, it is necessary to first adjust the thinking state.

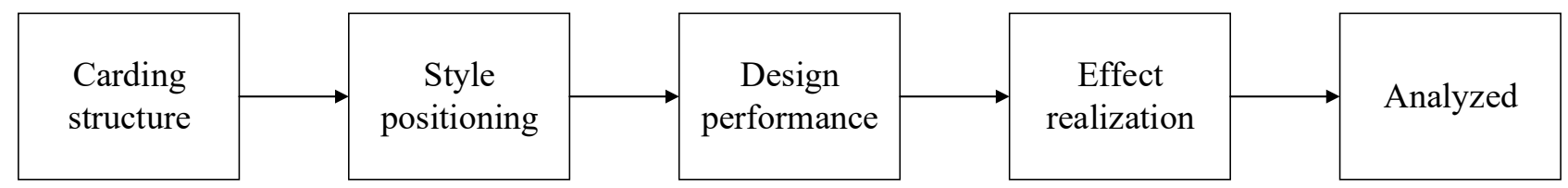

Figure 2. Layout design ideas

In terms of carding structure, sometimes, when the planner doesn't know how to better express the case, a manuscript like this will be received. First of all, the designer should have a set of design ideas and processes. When the design requirements for the production of $\mathrm{H} 5$ are obtained, it needs to make clear what the purpose of $\mathrm{H} 5$ is to do. It is supposed to have a good idea in minds, so that it is possible to make and conceive according to the purpose, and design the formal structure of each interface.

From the perspective of style positioning, it is to choose what kind of coat for H5. To choose a fresh and lovely style, or a mature and capable line, the key lies in which style is suitable for the people wearing clothes. Therefore, the use of hand-painted style of expression, or the use of tone elements to explain, mainly depends on what will be conveyed.

In view of effect realization, it includes vision, sound and interaction, which is a much more thoughtful part of the H5, because sensory synthesis is better than most good-looking single-screen works. There are many ways to achieve this, such as AE animation, or PS sequence frame. The key is to cooperate with the front-end to achieve the final effect. H5 is not a picture, but a combination of visual, auditory and interaction. Appropriate sound effects can be added to clever animation to make the whole experience more vivid and three-dimensional.

\section{Conclusion}

Using some research and analysis of visual psychology, the new media layout design rationally arranges the design elements, which will play a better role in human vision and arouse people's psychological resonance, namely the visual excitement mentioned above. In the design of new media format, it is necessary to deeply analyze the visual psychology and form our own concepts acted on the design of new media layout, so that it can make the design play a better role in communication.

From the visual psychological perspective, the new media layout design is profoundly analysed. It is pointed out that the correlation of visual psychology to the new media layout design coincides with future layout design development. Thus, it is pointed out to use diversity perspective of visual psychological to guide the practice of today's new media layout design. Through related analysis and summary of a series of visual psychology, how visual psychology is used in the new media layout design is analyzed, and the specific embodiment of visual psychology in the new media layout design is summed up. The excellent research on visual psychology at home and abroad is combined with the excellent practice cases of new media layout design to analyze. Finally, the thinking of visual psychology in new media layout design is proposed from three aspects: visual flow direction, visual information processing and visual spatial layout. 


\section{References}

[1]. Leno I J, Sankar S S, Ponnambalam S G. MIP model and elitist strategy hybrid GA-SA algorithm for layout design[J]. Journal of Intelligent Manufacturing, 2018:1-19.

[2]. Feng H, Xi L, Xia T, et al. Concurrent cell formation and layout design based on hybrid approaches[J]. Applied Soft Computing, 2018, 66:346-359.

[3]. Radcliffe J C, Page D, Naumann B, et al. Fifty Years of Water Sensitive Urban Design, Salisbury, South Australia[J]. Frontiers of Environmental Science \& Engineering, 2017, 11(4):7.

[4]. Giassi M, Göteman M. Layout design of wave energy parks by a genetic algorithm[J]. Ocean Engineering, 2018, 154:252-261.

[5]. Defersha F M, Hodiya A. A mathematical model and a parallel multiple search path simulated annealing for an integrated distributed layout design and machine cell formation[J]. Journal of Manufacturing Systems, 2017, 43:195-212.

[6]. Wu W, Fan L, Liu L, et al. MIQP-based Layout Design for Building Interiors[J]. Computer Graphics Forum, 2018, 37(2):511-521.

[7]. Guo Z, Li B. Evolutionary approach for spatial architecture layout design enhanced by an agentbased topology finding system[J]. Frontiers of Architectural Research, 2017, 6(1):53-62.

[8]. Arena P, Aubert J, Aiello G, et al. Thermal optimization of the Helium-Cooled Lithium Lead breeding zone layout design regarding TBR enhancement[J]. Fusion Engineering \& Design, 2017, 124. 